\title{
Anomalous drainage of inferior vena cava to left atrium in association with total anomalous pulmonary venous drainage
}

\author{
R K LAMB, S A QURESHI, R G PATEL, D I HAMILTON \\ From the Royal Liverpool Children's Hospital, Liverpool, and the Royal Manchester Children's Hospital, \\ Pendlebury, Manchester
}

\begin{abstract}
Drainage of the inferior vena cava directly into the left atrium is a rare but well recognised anomaly of systemic venous drainage. It has been reported in isolation and in association with other cardiac defects. We report a case of anomalous drainage of the inferior vena cava into the left atrium in association with total anomalous pulmonary venous drainage of the supracardiac type, a hitherto unreported combination.
\end{abstract}

\section{Case report}

A 7 month old boy presented with a three month history of intermittent cyanosis, breathlessness, and failure to thrive. $\mathbf{A}$ diagnosis of supracardiac total anomalous pulmonary venous drainage to the innominate vein was made at cardiac catheterisation and angiocardiography. The aortic saturation was $85 \%$ and the pulmonary artery pressure was $44 / 16$ $\mathrm{mm} \mathrm{Hg}$. The ratio of the right to the left ventricular peak systolic pressure was 0.63 .

Corrective surgery was performed through a left anterolateral thoracotomy with trans-sternal extension. A $12 \mathrm{~cm}$ common pulmonary venous channel was unobstructed throughout its course. The left atrium was larger than normally expected in this anomaly and there was a $12 \mathrm{~mm}$ secundum atrial septal defect. Cardiopulmonary bypass was established, a single systemic venous cannula being introduced through the right atrial appendage. Under circulatory arrest at $16^{\circ} \mathrm{C}$, with both venae cavae snared, the left atrium was opened posteriorly. The atrial septal defect was closed by direct suture and the common pulmonary venous channel was anastomosed to the left atrium. The ascending pulmonary vein was ligated. Postoperative progress was uneventful and he was discharged home.

Three months later his mother noticed cyanosis of the lips on exertion. At the age of 3 years, he was found to be centrally cyanosed with mild finger clubbing. Repeat cardiac catheterisation was performed via the right femoral vein. The catheter was manoeuvred easily from the inferior vena cava into the left atrium and with more difficulty into the superior vena cava. There was oximetric evidence of a significant right to left shunt, the left ventricular saturation being $89 \%$. The pulmonary artery pressure was $20 / 12$

Address for reprint requests: Dr S A Qureshi, Department of Paediatric Cardiology, Royal Liverpool Children's Hospital, Liverpool L77DG.

Accepted 20 January 1987 $\mathrm{mm} \mathrm{Hg}$ and the right to left ventricular systolic ratio was $0 \cdot 26$. A right atrial angiogram failed to opacify the inferior vena cava and there was no right to left shunting of the contrast medium (figure). Reoperation was considered necessary.

Through a median sternotomy cardiopulmonary bypass was established with bicaval cannulas. The inferior vena cava was cannulated directly as it could not be entered from the right atrium. The atrial appendages were morphologically normal. The azygos vein was not dilated. The right atrium was opened and the atrial septum was seen to be intact apart from a $2 \mathrm{~mm}$ defect within the previous repair. The coronary sinus was in the normal position, draining into the right atrium. The inferior vena caval orifice was not visible as it drained directly into the left atrium - to the left of the inferior portion of the interatrial septum. The atrial septum was excised and replaced by an oval patch of calf pericardium directing the inferior vena cava into the right atrium.

The boy has made a satisfactory recovery and three months after operation is symptom free.

\section{Discussion}

Anomalies of systemic venous drainage have been reported extensively but drainage of the inferior vena cava directly into the left atrium is rare. It has been reported in isolation on three occasions ${ }^{1-3}$ and has recently been described in association with a secundum atrial septal defect and hemianomalous pulmonary venous drainage in one patient and with tetralogy of Fallot in another. ${ }^{4}$ It has also been reported in association with multiple pulmonary arteriovenous fistulas. ${ }^{3}$ The diagnosis may be obscured by the presence of other cardiac defects. One of the cases described by Sanchez and Human highlights the problem. In this patient, it was recognised only at repeat cardiac catheterisation, performed because of cyanosis after total repair of Fallot's tetralogy. In our case the patient had previously undergone redirection of supracardiac total anomalous pulmonary venous drainage. Because only one venous cannula was introduced into the right atrium to establish cardiopulmonary bypass the anomalous drainage of the inferior vena cava was not suspected. Caval snares obscured the orifice of the inferior vena cava in the left atrium. Visualisation of this might have been easier had snares not been used.

Functional drainage of the inferior vena cava into the left atrium has also been reported. This has been attributed to 

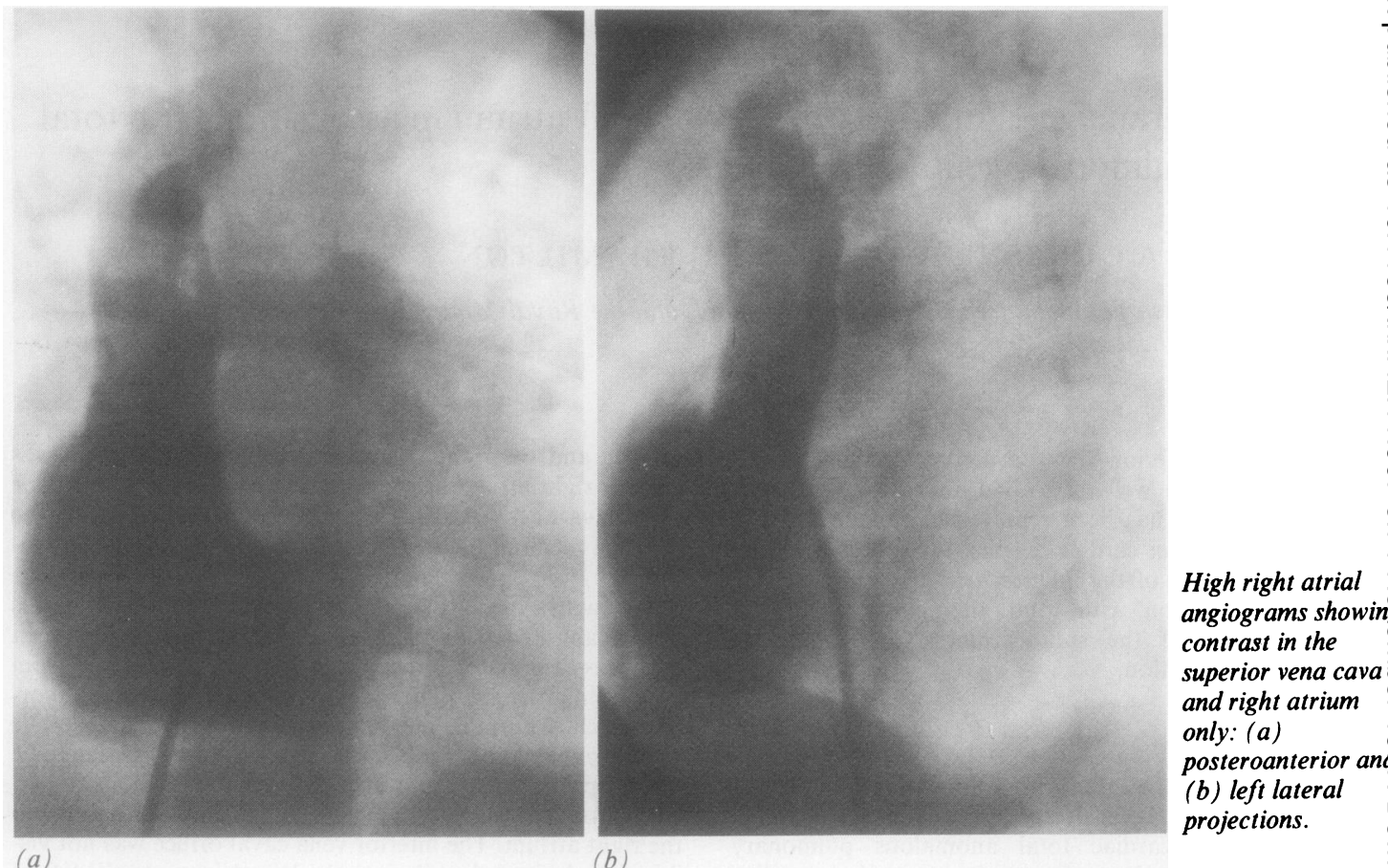

(a)

the combination of a hypertrophied Eustachian valve and a low secundum atrial septal defect. ${ }^{6}$ Surgical closure of a secundum atrial septal defect may rarely result in similar drainage of the inferior vana cava if the Eustachian valve is incorporated in the repair. ${ }^{7}$

\section{References}

1 Gardner DL, Cole L. Long survival with inferior vena cava draining into left atrium. Br Heart $J$ 1955;17:93-7.

2 Venables AW. Isolated drainage of the inferior vena cava to the left atrium. Br Heart J 1963;25:545-8.

3 Meadows WR, Bergstrand I, Sharp JT. Isolated anomalous connection of a great vein to the left atrium: the syndrome of cyanosis and clubbing, "normal" heart, and left ventricula hypertrophy on electrocardiogram. Circulation 1961;2 669-76.

4 Sanchez HE, Human DG. Drainage of the inferior vena cava to the left atrium. Ped Cardiol 1986;6:207-9.

5 Black H, Smith GT, Goodale WT. Anomalous inferior vena cav draining into the left atrium associated with intact interatriat septum and multiple pulmonary arteriovenous fistulac. Circulation 1964;29:258-67.

6 Gallacher ME, Sperling DR, Gwinn JL, Meyer BW, Fyler DC్ Functional drainage of the inferior vena cava into the lef atrium: three cases. Am J Cardiol 1963;12:561-6.

7 Mustard WT, Firor WB, Kidd L. Diversion of the venae cavă into the left atrium during closure of atrial septal defects. Thorac Cardiovasc Surg 1964;47:317-24. 